\title{
Dysplasia epiphysialis punctata with ocular anomalies
}

\section{A. HAMMOND}

From the Departments of Ophthalmology and Paediatrics, Free University, Amsterdam

Dysplasia epiphysialis punctata was first described by Conradi (I9I4), who called the condition chondrodystrophia foetalis hypoplastica. The diagnosis is made on the radiological appearance of characteristic stippling or punctuate calcification in the region of the epiphyseal centres. The skeletal changes may be accompanied by involvement of the cardiovascular and central nervous systems and of the skin and eyes. In the present case, in addition to the cataracts which are the characteristic ocular features, there were anomalies of the angles of the anterior chambers, a complication which has not previously been reported in association with this condition.

\section{Case report}

The propositus was the sixth child of healthy unrelated parents. The pregnancy was uncomplicated and the baby was born prematurely at 37 weeks, weighing 2,820 g. and measuring $45 \mathrm{~cm}$. long.

\section{General examination (Fig. I)}

The typical saddle nose and shortening of the proximal parts of the extremities gave the baby an achondroplastic appearance. The flexed hip-joint could not be extended. The fingers and toes also showed a flexion contracture, and the second finger and toe were larger than the third finger and toe. Both hands had a simian line. There was a cleft soft palate. No cutaneous manifestations were encountered. Post partum a loud systolic murmur developed.

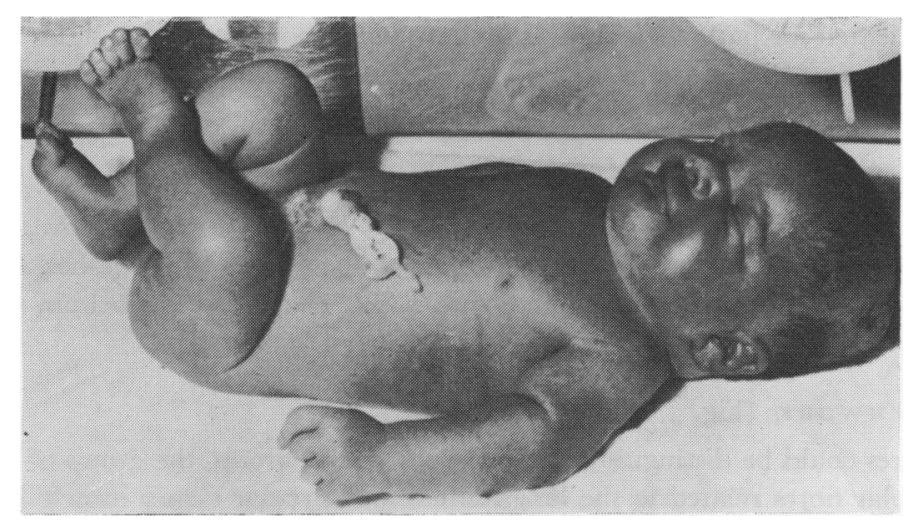

FIG. I Clinical appearance 
Radiology (Fig. 2)

$X$ rays of the shoulder, hip, and knee joints showed typical stippling of the epiphyses. The distal parts of the femur and humerus showed typical mushrooming. The skull was normal.

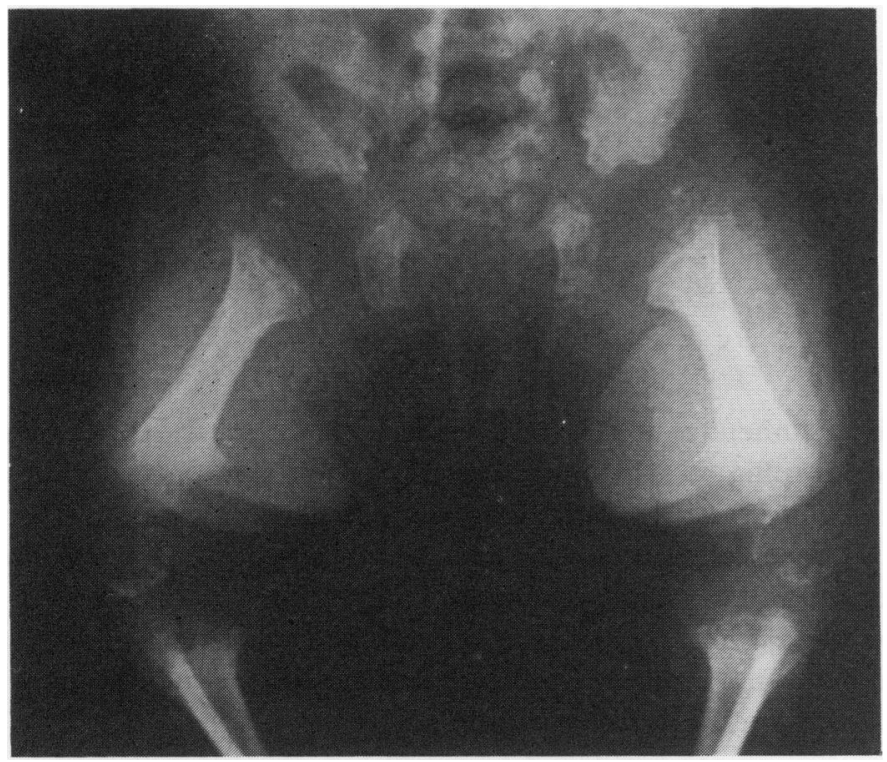

FIG. 2 Radiographic appearance, showing stippled epiphyses

Chromosomal investigation

A large satellite was present on one of the chromosomes, Denver group IV (1 $3 /$ I $\left._{5}\right)$. The sex chromosomes were $\mathrm{XX}$.

\section{Ophthalmological findings}

Examination of the eyes showed that both were equally large with slightly hazy corneae. The ocular tensions seemed elevated on palpation. Both eyes had a cataract. After the use of pilocarpine the corneae cleared. A posterior embryotoxon was present. The irides were atrophic.

\section{Termination}

After 2 months the child died of pneumonia and a congenital heart defect (a patent foramen ovale and a patent ductus arteriosus).

\section{Pathological examination}

The eyes were enucleated for pathological investigation, and the sections were stained with haematoxylin and eosin and Van Gieson stains.

\section{GORNEA}

The corneal epithelium, stroma, and Descemet's membrane were normal. There was a prominent ring of Schwalbe in which Descemet's membrane ended. The ring itself was covered by a single layer of endothelium. From the prominent line of Schwalbe a very broad adhesion ran towards the iris root. It consisted of iris stroma, lined with a single layer of epithelium-the posterior embryotoxon.

TRABEGULAR MESHWORK (Fig. 3, opposite).

Three groups of fibres could be distinguished: the corneo-scleral group, the group of fibres built up of the uveal trabecular fibres related to the longitudinal and circular ciliary muscle fibres, and the 


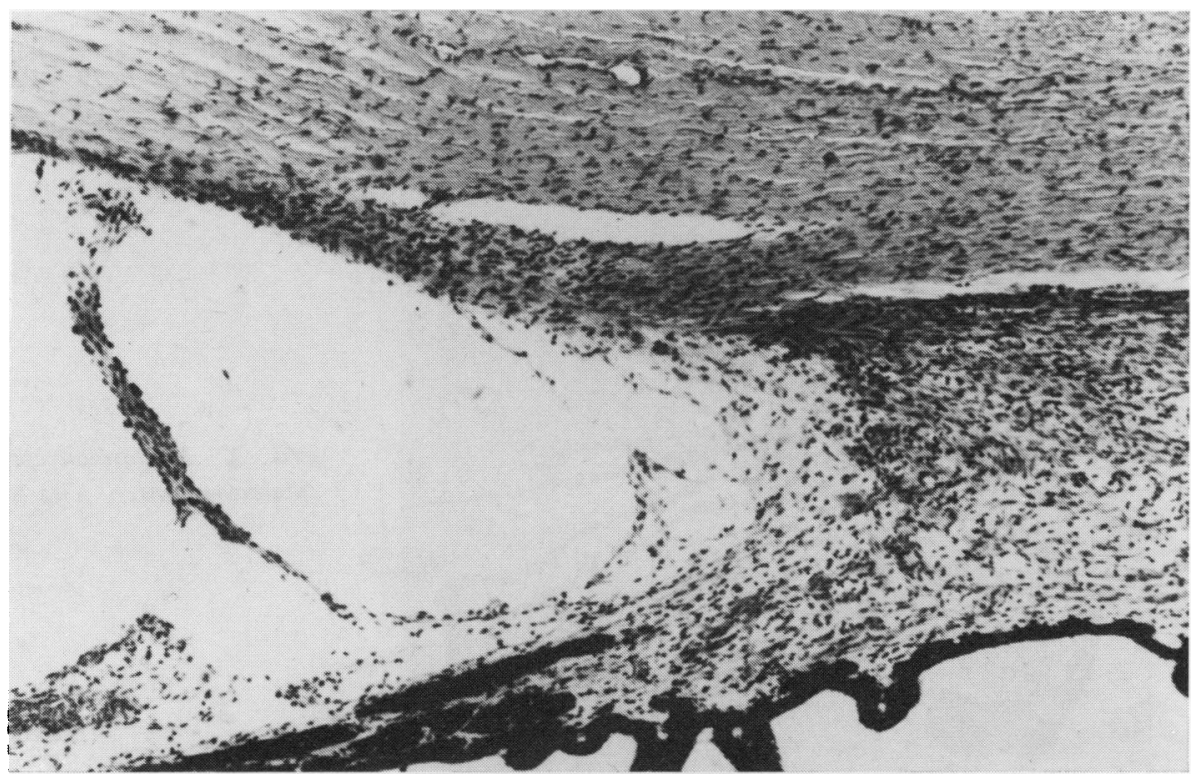

FIG. 3 Microscopic appearance of the angle of the anterior chamber. $\times 94.5$

group consisting of the fibres of the uveal meshwork relating to the root of the iris, the anterior surface of which is situated on the endothelial surface of the edge of Descemet's membrane; it covers the anterior border ring of Schwalbe, the posterior termination of which lies in the most superficial layers of the iris stroma.

IRIS

The iris stroma was markedly hypoplastic but, considering the age of the child, this could be accepted as normal. The sphincter pupillae and the pigment layer were normally developed. There was a lobulation of the peripheral boundary of the pigment layer.

CILIARY BODY

The meridional ciliary muscle was well represented, while the circular fibres were still poorly developed. The scleral spur was not fully developed, and the canal of Schlemm could be seen anterior to it. The ciliary processes reached up to one-third of the iris and were atrophic.

LENS

Peripherally the epithelium was normal. The lens fibres at the equator were well developed. The posterior part of the lens contained some swollen cells. The anterior part of the lens consisted of a homogeneous substance without any structures (Fig. 4, overleaf).

VITREOUS BODY This showed no anomalies.

GHOROID AND RETINA These were well developed.

OPTIC NERVE This had a slightly cupped disc, which could be considered as normal.

\section{Discussion}

Anomalies of the angle of the anterior chamber occur frequently in various systemic connective tissue disorders (Osgood-Schlatter's disease, Perthes' disease, the Marchesani 


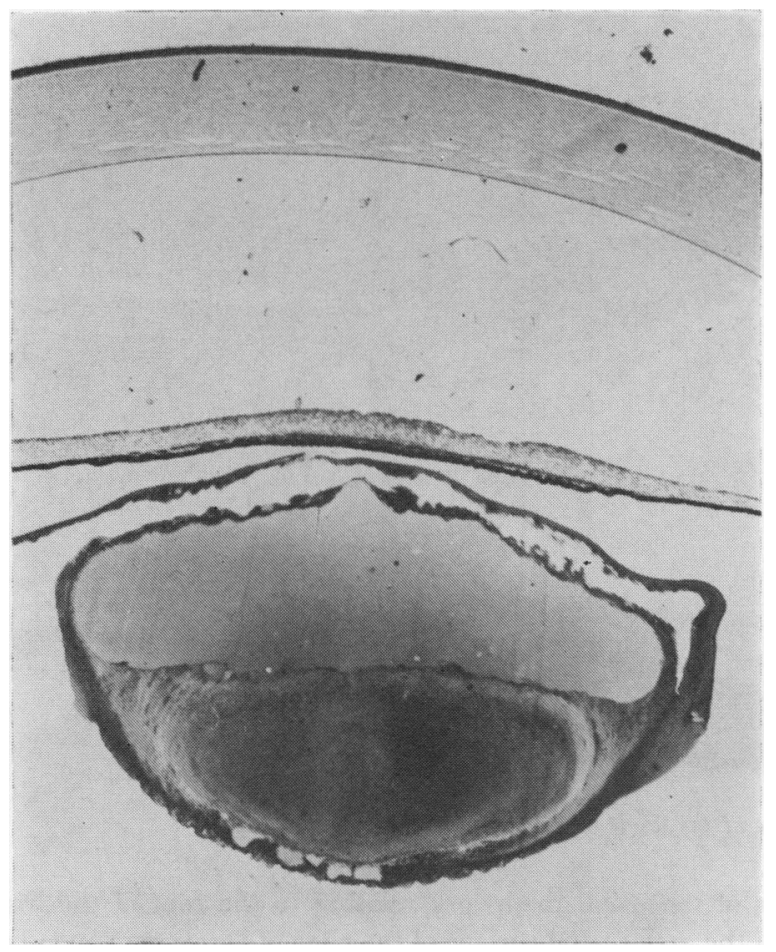

FIG. 4 Microscopic appearance of the cataractous lens. $\quad \times 94.5$

syndrome, and the oculo-dento-digital dysplasia syndrome) (François, 196ı; Alkemade, I969; Burian, von Noorden, and Ponseti, I96o).

Anomalies of the anterior chamber angle have not been described before in dysplasia epiphysialis punctata, the only ocular anomalies reported previously being cataract and optic atrophy. The embryological development of the angle is a very complicated process and it seems possible that the development is chromosomally determined and linked with the mesodermal development of the skeleton of the human being. Biochemical "derangements" could be possible agents in the anomalies of the angle, where three major tissues come together in a very small space.

\section{Summary}

A case of dysplasia epiphysialis punctata has been described together with its ocular anomalies. In both eyes there was a cataract and a prominent line of Schwalbe, together with adhesions between iris and cornea in the region of the ring of Schwalbe (embryotoxon posterior). There was also hypoplasia of the iris. Excluding the cataract, these changes are similar to those of dysgenesis mesodermalis corneae et iridis (Rieger's syndrome).

Glaucoma was diagnosed clinically by the hazy corneae which cleared after treatment with pilocarpine. The diagnosis could not be verified by measurement.

\section{References}

alkemade, P. P. H. (1969) "Dysgenesis Mesodermalis of the Iris and the Cornea". Van Gorcum, Assen, Holland

BURIAN, H. M., von NOORDEN, G. K., and PONSETI, I. v. (1960) Arch. Ophthal. (Chicago), 64, 67 I

CONRADI, E. (1914) Jb. Kinderheilk., 80, 86

FRANÇOIs, J. (1961) Amer. F. Ophthal., 52, 207 